\title{
Post-traumatic Winking Induced by Extension of the Hand: A Case Report of a Novel Synkinesis
}

Riet Vangheluwe $^{\mathrm{a}}$, Linda Postma ${ }^{\mathrm{b}}$, Marcel Ten Tusscher ${ }^{\mathrm{c}}$, Laura WJ Baijens ${ }^{\mathrm{d}}$, Robert van Oostenbrugge ${ }^{\mathrm{a}}$

${ }^{a}$ Department of Neurology, Maastricht University Medical Center, Maastricht, The Netherlands

${ }^{b}$ Department of Radiology, Maastricht University Medical Center, Maastricht, The Netherlands

${ }^{c}$ Department of Ophthalmology, University Hospital of Antwerp, Antwerp, Belgium

${ }^{\mathrm{d}}$ Department of Otorhinolaryngology, Head and Neck Surgery, Maastricht University Medical Center, Maastricht, The Netherlands

\begin{abstract}
:
Several synkinesis syndromes have been reported in the literature. Synkinesis syndromes are rare and are most commonly congenital or follow post-traumatic reinnervation. We describe a novel synkinesis syndrome that developed several months after cervical spinal cord infarction due to a herniated disc in a 29-year-old woman. When the patient overstretched the extensor muscles of the right hand, the right upper eyelid raised automatically and nasal congestion developed. We hypothesize that aberrant reinnervation of the intermediolateral columns of the spinal cord at level C8-T2 by motor neurons of the extensor muscles of the hand occurred.
\end{abstract}

Keywords: Synkinesis, winking, hand, reinnervation, extensor muscles

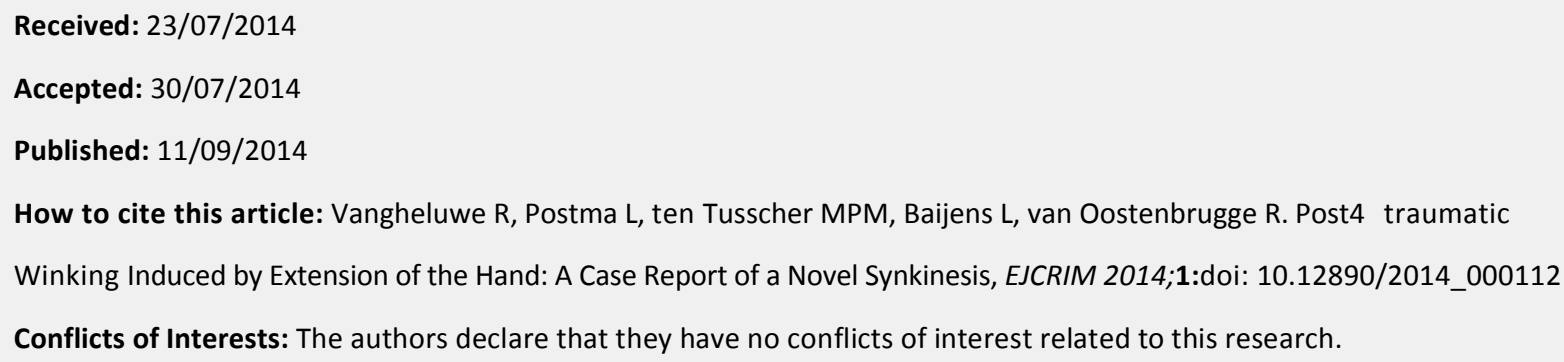




\section{Case report}

A 29-year-old woman without a medical history or previous trauma developed progressive painless weakness and paraesthesias in both arms, quickly followed by weakness in both legs and loss of sensation in the trunk. Shortly afterwards she developed progressive respiratory insufficiency which necessitated mechanical ventilation.

The differential diagnosis at the time was Guillain-Barré syndrome, autoimmune myelitis or cervical disc herniation with myelopathy. During history taking, the patient mentioned that the symptoms started with pain in the neck during a game of tennis. Neurological clinical examination revealed a slight weakness proximally in both arms (MRC grade 4) and severe weakness (MRC grade 0-2) in the distal hand musculature and triceps muscles. The left leg was paralysed and the right leg was seriously weakened (MRC grade 3 proximally and grade 2 distally). She had a hypaesthesia at and below dermatome C6, a sensory level on the trunk at Th4 and hypaesthesia of both legs. Vibration and joint position sense were intact. The triceps, patellar and Achilles tendon reflexes were absent (grade 0). Eliciting the plantar reflex resulted in a triple response bilaterally.

Under the assumption of an anterior spinal artery syndrome, an MRI of the cervical spine was performed. Sagittal T2-weighted images (Fig. 1a) showed a herniated disc at level C5-C6 and hyperintensity of the anterior part of the spinal cord from C4 to Th1 with swelling and diffusion restriction (Fig. 1C). Post-contrast images showed enhancement of this region. The findings were consistent with infarction of the spinal cord in the distribution of the anterior spinal artery. A spinal MRA, later confirmed by digital subtraction angiography, revealed a hypoplastic right vertebral artery, without signs of dissection or vasculitis. The anterior spinal artery was not visualized at level C3-C8.
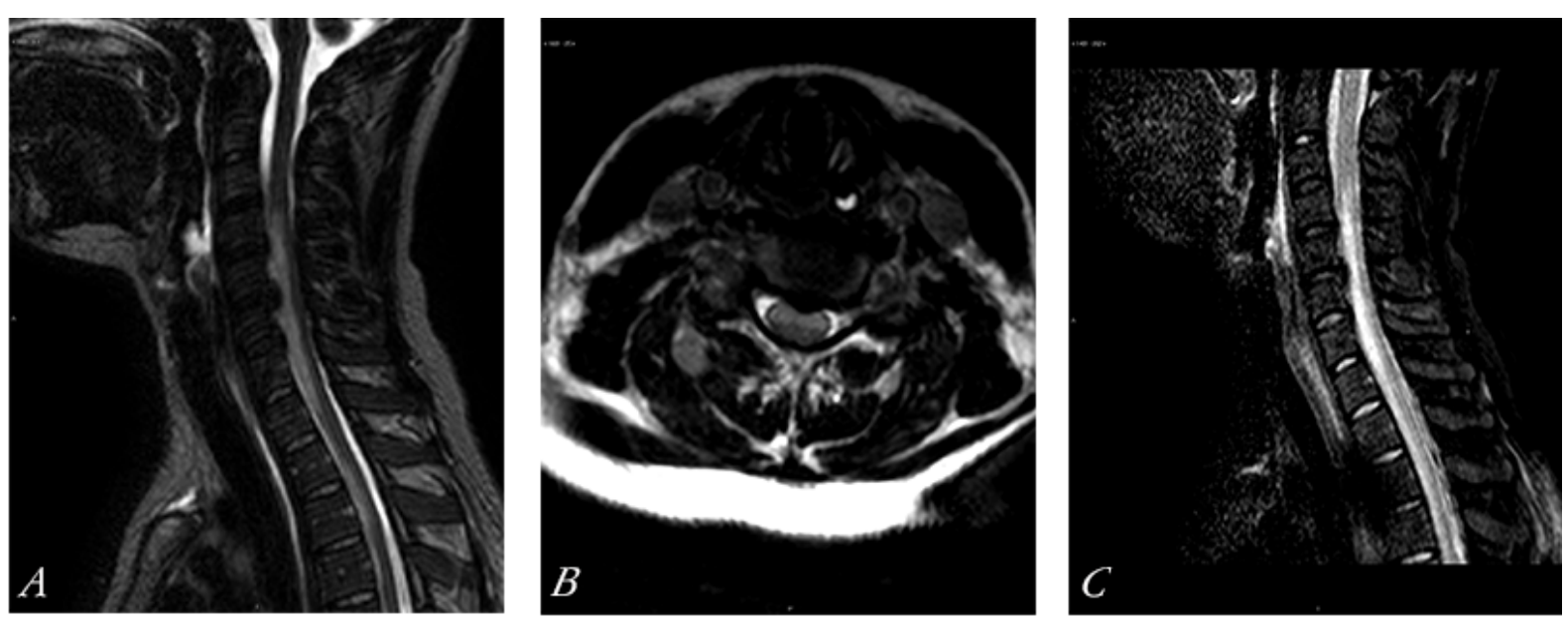

Figure 1 a-c: MRI of the cervical spinal cord (a: sagittal T2; b: axial T2; c: diffusion weighted imaging) showing a herniated disc at C5-C6, a hyperintense spinal cord at C4-Th1, and swelling and diffusion restriction. 
Based on the radiological examinations, a diagnosis of subacute occlusion of the anterior spinal artery due to a herniated disk at $\mathrm{C} 5-\mathrm{C} 6$ was made. No autoimmune disease or underlying clotting disorder was found and cardiac embolism was ruled out. Surgical intervention was withheld because the extensive infarction of the spinal cord was presumed irreversible and there was a high risk of further damaging the spinal cord during surgery.

After a few weeks spastic tetraparesis with slight hypaesthesia remained, and the patient needed help eating and defecating and received intermittent catheterisation. After revalidation she regained most of the power in her legs and left arm. Weakness of the right arm remained.

Nine months later, a follow-up MRI of the cervical spinal cord showed bulging of the disc at level C5C6 with distortion of the spinal cord. The anterior spinal cord was atrophic with a so-called snake-eye appearance, consistent with late sequela of anterior spinal cord ischaemia (Fig. 2)
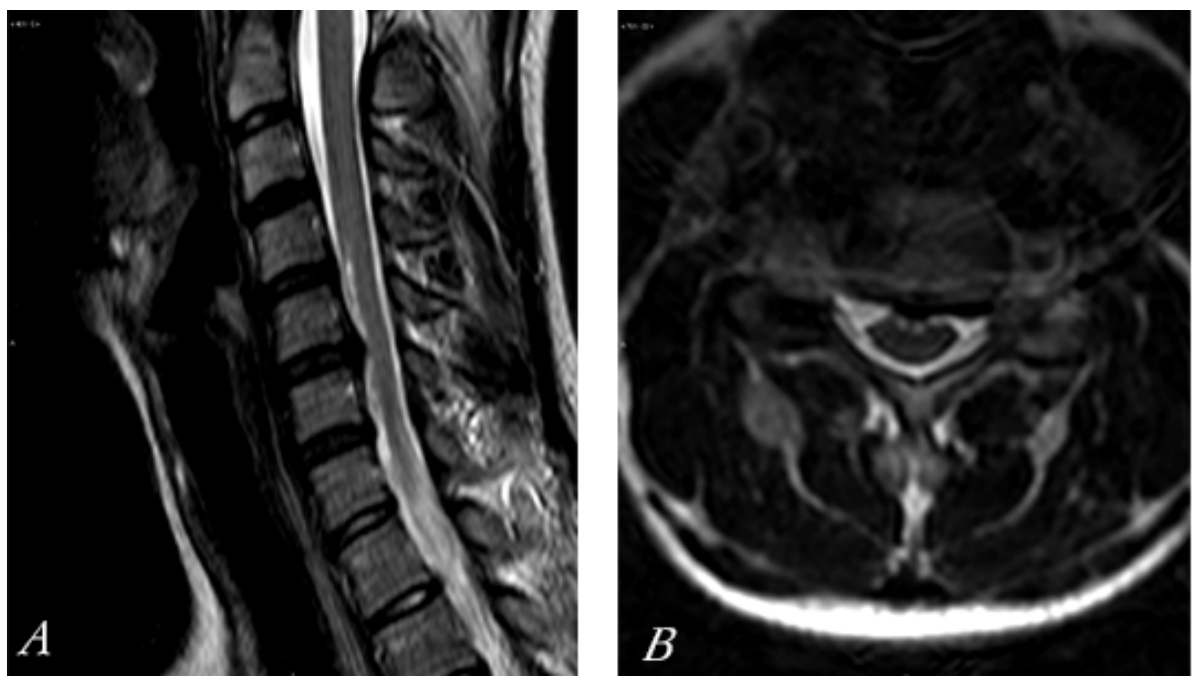

Figure $2 a, b$ : MRI of the cervical spinal cord (a: sagittal T2; b: axial T2) showing atrophic anterior spinal cord with a snake-eye appearance

At neurological follow up 2.5 years after the onset of symptoms, the patient complained of involuntary raising of the right eyelid when passively overstretching the extensor muscles of the right hand. This was combined with paroxysmal stuffiness in the nose. Synkinesia was suspected as a result of neurological regeneration. The patient was referred to an otolaryngologist and an ophthalmologist to determine the exact location of the aberrant reinnervation.

At ophthalmological investigation, both eyes showed very slow dilation and a lack of mydriasis in the dark. The pupils did not dilate after instillation of cocaine $5 \%$, but mydriasis of both pupils occurred after local administration of amphetamine $1 \%$ in both eyes. These results indicated a sympathetic lesion central to the superior cervical ganglion.

Otoscopic examination was normal, while nasendoscopy showed bilateral very congestive inferior conchae. An inhalation allergy was excluded after a negative phadiatop test. 


\section{Discussion}

The pre-ganglionic Horner syndrome of both eyes revealed by ophthalmological testing was presumably caused by damage to the ciliospinal centre of Budge and Waller, located in the intermediolateral columns of the spinal cord at level C8-T2.[1]

The sympathetic innervation of the eye exits at level C5-T4, from where the sympathetic fibres continue to the superior cervical ganglion at the bifurcation of the carotid artery and innervate Muller's muscle. This muscle contributes to the over-elevation of the eyelid when a patient becomes excited or fearful and leads to mild ptosis when dysfunctional. A cervical hernia can thus cause a sympathetic disorder in the eye. Horner syndrome due to cervicothoracic syringomyelia has been described previously.[2] The mildness of the ptosis is explained by an intact oculomotor innervated superior levator palpebrae muscle which is the most important muscle elevating the eyelid.

Furthermore, the damaged intermediolateral columns of the spinal cord presumably also caused the autonomic innervation of the nasal cycle to be disturbed, resulting in congestion of the nasal conchae.

In the literature, aberrant innervation causing synkinesis is most often described as part of congenital aberrant innervation syndromes (e.g. Marcus Gunn syndrome,[3] Marin-Amat syndrome [1] and trigemino-abducens synkinesis[4]). Post-traumatic and post-operative syndromes have also been reported (e.g. the breathing arm).[5] We now add another, new form of synkinesis composed of involuntary raising of the right upper eyelid and nasal congestion when overstretching the extensor muscles of the right hand. It developed after recovery from an anterior spinal artery syndrome causing severe quadriparesis. The underlying cause of the anterior spinal artery syndrome was most likely disc herniation at C5-C6 with compression of the myelum causing ischaemia. During recovery, synkinesis must have occurred between the intermediolateral columns (innervating Muller's muscle) and the extensor muscles of the right forearm.

\section{Learning Points}

- Aberrant innervation causing synkinesis can develop after an anterior spinal artery syndrome.

- Pre-ganglionic Horner syndrome of both eyes can be caused by damage to the ciliospinal centre of Budge and Waller, located in the intermediolateral columns of the spinal cord at level C8-T2.

- Damage to the intermediolateral columns of the spinal cord can presumably cause the autonomic innervation of the nasal cycle to be disturbed resulting in congestion of the nasal conchae. 


\section{European Journal \\ of Case Reports in \\ Internal Medicine}

\section{References}

1. Pavone P, Garozzo R, Trifiletti RR. Marin-Amat syndrome: case report and review of the literature. J Child Neurol 1999;14:266-268.

2. Cagan E, Sayin R, Doğan M, Peker E, Cağan HH, Caksen H. Bilateral brachial plexus palsy and right Horner syndrome due to congenital cervicothoracal syringomyelia. Brain Dev 2010;32:595-597.

3. Kodsi M. Marcus Gunn jaw winking with trigemino-abducens synkinesis. J AAPOS 2000;4:316-317.

4. Lai T, Chen C, Selva D. Bilateral congenital trigemino-abducens synkinesis. Arch Ophthalmol 2003;121:1796-1797.

5. Lam L, Engstrom J. Teaching video Neurolmages: the breathing arm: respiratory brachial synkinesis. Neurology 2010;74:e69. 